\title{
Desempenho agronômico de pimenta dedo de moça sob adubação orgânica e mineral
}

\author{
Agronomic performance of girl finger pepper under organic and mineral fertilization \\ Desempeño agronómico del pimiento dedo de niña bajo fertilización orgánica y mineral
}

Recebido: 14/03/2021 | Revisado: 21/03/2021 | Aceito: 23/03/2021 | Publicado: 01/04/2021

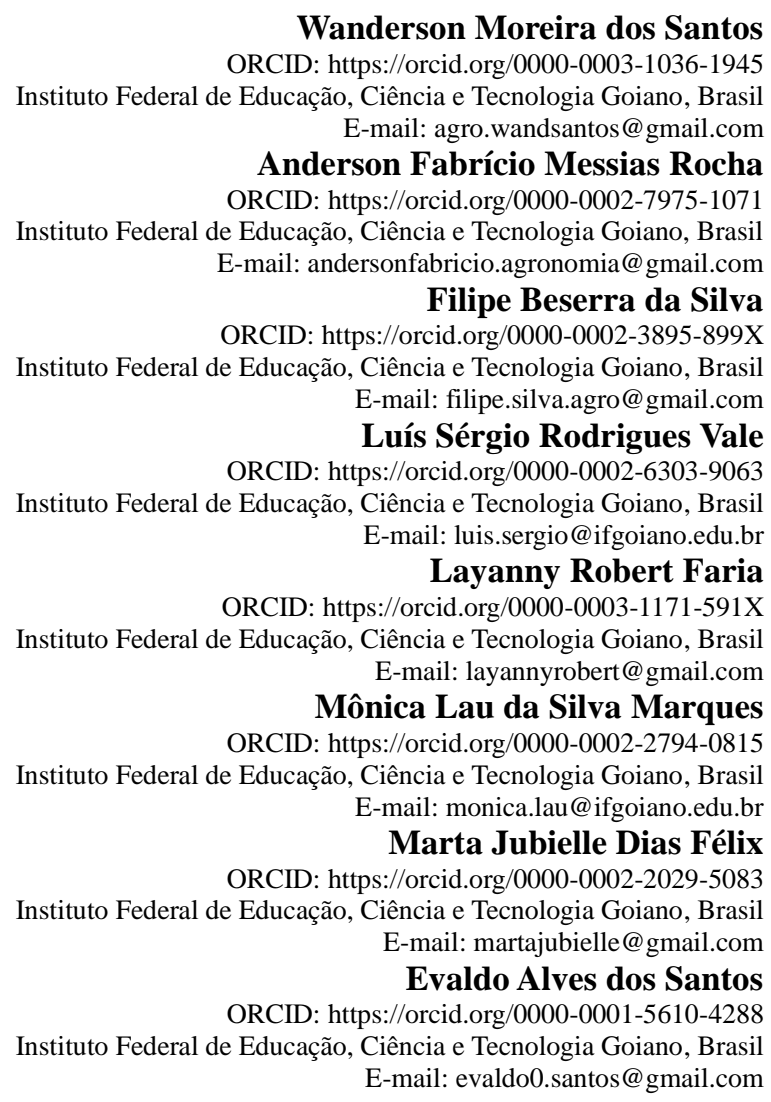

\section{Resumo}

O cultivo de pimentas é realizado basicamente em todo território brasileiro, principalmente, pela agricultura familiar. $\mathrm{O}$ uso de alternativas para baratear o processo de produção, em particular no uso de insumos, é uma alternativa que pode proporcionar maior lucratividade para o agricultor. Dessa forma, objetivou-se com este experimento avaliar a qualidade agronômica da produção de pimenta dedo de moça sob diferentes doses de adubo orgânico e mineral. O delineamento experimental foi em blocos casualizados, com seis tratamentos e quatro repetições. Os tratamentos foram as doses de adubo orgânico: $0,15,30,45$ e $60 \mathrm{t} \mathrm{ha}^{-1}$ de esterco bovino e um tratamento com adubação mineral. Foi avaliado o desempenho agronômico da cultura. As doses de adubo orgânico não proporcionaram diferença significativa na produtividade e no número de frutos por planta de pimentas. Os resultados foram maiores que no tratamento controle. A doses de adubo orgânico de 45 e $60 \mathrm{t} \mathrm{ha}^{-1}$ proporcionaram maiores produtividades de pimentas que o controle. As doses de adubo orgânico e mineral não proporcionaram diferença significativa nas variáveis de produção, comprimento e diâmetro de frutos e espessura da polpa. O maior índice de eficiência agronômica quanto à produtividade foi obtido na dose de $45 \mathrm{t} \mathrm{ha}^{-1}$ de adubo orgânico em relação ao adubo mineral. $\mathrm{O}$ uso de esterco bovino pode ser indicado na produção de pimenta dedo de moça na região de Ceres, GO.

Palavras-chave: Capsicum baccatum; Esterco bovino; Produção orgânica; Cultivo em sequeiro.

\begin{abstract}
The cultivation of peppers is carried out basically throughout the Brazilian territory, mainly by family farming. The use of alternatives to cheapen the production process, particularly in the use of inputs, is an alternative that can provide greater profitability for the farmer. Thus, the objective of this experiment was to evaluate the agronomic quality of the production of girl finger pepper under different doses of organic fertilizer and mineral fertilizer. The experimental design was in randomized blocks, with six treatments and four replications. The treatments were the
\end{abstract}


doses of organic fertilizer: $0,15,30,45$ and $60 \mathrm{t} \mathrm{ha}^{-1}$ of bovine manure and a treatment with mineral fertilization. The agronomic performance of the crop was evaluated. The doses of organic fertilizer did not provide significant difference in productivity and in the number of fruits per pepper plant. The results were greater than in the control treatment. The doses of organic fertilizer of 45 and $60 \mathrm{t} \mathrm{ha}^{-1}$ provided higher yields of peppers than the control. The doses of organic and mineral fertilizer did not provide significant difference in the variables of production, length and diameter of fruits and pulp thickness. The highest index of agronomic efficiency in terms of productivity was obtained at a dose of $45 \mathrm{t} \mathrm{ha}^{-1}$ of organic fertilizer in relation to mineral fertilizer. The use of bovine manure can be indicated in the production of paprika pepper in the region of Ceres, GO.

Keywords: Capsicum baccatum; Cattle manure; Organic production; Rainfed cultivation.

\section{Resumen}

El cultivo de pimientos se realiza básicamente en todo el territorio brasileño, principalmente por agricultura familiar. El uso de alternativas para abaratar el proceso productivo, particularmente en el uso de insumos, es una alternativa que puede brindar mayor rentabilidad al agricultor. Así, el objetivo de este experimento fue evaluar la calidad agronómica de la producción de pimiento dedo de niña bajo diferentes dosis de fertilizante orgánico y fertilizante mineral. El diseño experimental fue en bloques al azar, con seis tratamientos y cuatro repeticiones. Los tratamientos fueron las dosis de fertilizante orgánico: $0,15,30,45$ y $60 \mathrm{t} \mathrm{ha}^{-1}$ de estiércol bovino y un tratamiento con fertilización mineral. Se evaluó el comportamiento agronómico del cultivo. Las dosis de fertilizante orgánico no proporcionaron diferencia significativa en la productividad y en el número de frutos por planta de pimiento. Los resultados fueron mayores que en el tratamiento control. Dosis de fertilizante orgánico de 45 y $60 \mathrm{t} \mathrm{ha} \mathrm{ha}^{-1}$ proporcionaron mayores rendimientos de pimiento que el testigo. Las dosis de fertilizante orgánico de 45 y $60 \mathrm{t} \mathrm{ha}^{-1}$ proporcionaron mayores rendimientos de pimiento que el testigo. Las dosis de fertilizante orgánico y mineral no proporcionaron diferencia significativa en las variables de producción, longitud y diámetro de frutos y espesor de pulpa. El mayor índice de eficiencia agronómica en términos de productividad se obtuvo con una dosis de $45 \mathrm{t} \mathrm{ha}^{-1}$ de fertilizante orgánico en relación al fertilizante mineral. El uso de estiércol bovino puede estar indicado en la producción de ají en la región de Ceres, GO.

Palabras clave: Capsicum baccatum; Estiércol de ganado; Produccion organica; Cultivo de secano.

\section{Introdução}

As pimentas são as espécies e variedades do gênero Capsicum com frutos geralmente menores que os pimentões, com diferentes formatos, frequentemente de paladar pungente, embora existam pimentas doces (Carvalho et al., 2003). No Brasil as pimentas do gênero Capsicum são consumidas e comercializadas em forma de conservas, molhos, in natura e desidratadas (Freitas et al., 2008).

O gênero Capsicum possui cerca de 25 espécies, sendo cinco domesticadas: $C$. annuum L. var. annuum (pimentão), $C$. baccatum L. var. pendulum (Pimenta dedo de moça), C. chinense Jacq. (Pimenta de cheiro), C. frutescens L. (Pimenta malagueta) e $C$. pubescens (Pimenta rocoto), e as demais espécies são semidomesticadas e silvestres (Reifschneider, 2000).

O cultivo de pimentas no Brasil é considerado patrimônio da agrobiodiversidade nacional, sendo segmento de mercado desde pequenas agroindústrias até a exportação por empresas multinacionais, com forte expressão tanto na indústria alimentícia, quanto na farmacêutica e cosmética (Rodrigues, 2016).

O cultivo de pimentas ocorre praticamente em todas as regiões do país e é um dos melhores exemplos de integração entre a agricultura familiar e a agroindústria (Costa \& Henz, 2007). Alternativas de baratear o processo de produção, em particular no uso de insumos, são meios que podem proporcionar maior lucratividade para o agricultor, e a utilização de adubos orgânicos como o esterco de ovino, bovino, suíno e cama de frango é uma opção importante para diminuir os custos de produção (Vazquez et al., 2010). Além disso, esses materiais proporcionam benefícios de melhoria da atividade biológica, física, química e o aumento do teor de carbono no solo (Zavattaro et al., 2017).

A demanda por produtos cultivados em sistema orgânico é uma tendência crescente do mercado de hortaliças e um processo em expansão. Nesse sentido tem-se buscado a ampliação de pesquisas científicas que contribua para desenvolver novas técnicas, insumos, manejos e tecnologias para esse modelo de produção (Dias et al., 2015; Ponisio et al., 2014). Assim, estudos com esterco tem mostrado que o uso adequado pode aumentar a produtividade das culturas além de ser economicamente viável (Oliveira et al., 2013; Silva et al., 2019; Zavattaro, 2017). É, portanto, uma opção na agricultura, 
principalmente pelos agricultores familiares. Ainda são poucos os estudos de respostas à adubação orgânica para a cultura de pimentas do gênero Capsicum, em particular na região Central do Brasil.

Dessa forma, objetivou-se com este experimento avaliar a qualidade da produção de pimenta dedo de moça sob diferentes doses de adubo orgânico e mineral.

\section{Metodologia}

O experimento foi realizado no campo experimental do Instituto Federal Goiano - Campus Ceres, no município de Ceres, GO, de janeiro a agosto de 2017. Os dados de precipitação pluviométrica e temperatura média no período encontram-se na Figura 1. Esses dados foram obtidos próximo à área experimental, na estação meteorológica do Instituto Federal Goiano Campus Ceres, Ceres GO.

Figura 1. Precipitação e temperatura média mensal nos meses de janeiro a agosto de 2017.

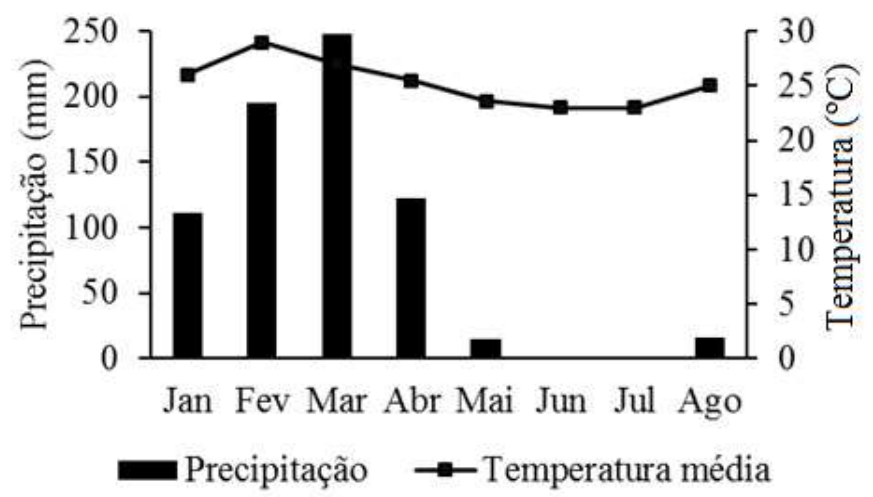

Fonte: Autores.

O solo da área experimental foi classificado como Latossolo vermelho (Embrapa, 2013), com as seguintes características química e física de acordo com a análise de solo, na profundidade de 0-20 cm: $438 \mathrm{~g} \mathrm{~kg}^{-1}$ de Argila; $523 \mathrm{~g} \mathrm{~kg}^{-1}$ de Areia e $39 \mathrm{~g} \mathrm{~kg}^{-1}$ de Silte; $\mathrm{pH}$ em água (1:25) 6,41; 69,45 $\mathrm{mg} \mathrm{dm}^{-3} \mathrm{de} \mathrm{P}$ (Mehlich); 145,5 $\mathrm{mg} \mathrm{dm}^{-3} \mathrm{de} \mathrm{K}^{+}$; 3,36 Cmolc $\mathrm{dm}^{-3}$ de $\mathrm{Ca}^{2+;} 1,1 \mathrm{Cmolc} \mathrm{dm}^{-3} \mathrm{de} \mathrm{Mg}^{2+} ; 1,5 \mathrm{Cmolc} \mathrm{dm}^{-3} \mathrm{de} \mathrm{H}+\mathrm{Al} ; 76,2 \%$ de saturação por bases (V\%) e 22,2 $\mathrm{g} \mathrm{dm}^{-3}$ de matéria orgânica.

As mudas de pimentas foram produzidas em casa de vegetação com substrato comercial Plantmax ${ }^{\circledR}$ e em embalagens descartáveis de $200 \mathrm{~mL}$. As mudas foram transplantadas a campo quando apresentaram de 6 a 12 folhas definitivas, cerca de 60 dias após a semeadura. A área foi preparada de forma convencional, com uma aração e duas gradagens e o plantio manual.

O delineamento experimental foi em blocos casualizados e seis tratamentos, com cinco doses de adubo orgânico e um tratamento com adubo mineral. Os tratamentos com as doses de adubo orgânico foram: 0, 15, 30, 45 e $60 \mathrm{t} \mathrm{ha}^{-1}$ de esterco bovino e um tratamento com adubação mineral $\mathrm{O}$ tratamento Zero (0) foi o Controle. Foram utilizadas quatro repetições $\mathrm{O}$ esterco bovino passou pelo processo de curtimento, que é o envelhecimento do esterco sob condições naturais, não controladas (Souza \& Alcântara, 2008). O adubo orgânico foi aplicado na cova 15 dias antes do plantio e apresentou os seguintes resultados, com base na matéria seca: $\mathrm{pH}$ em água de 8,65; 4,94 Cmolc dm-3 de $\mathrm{Ca}^{2+} ; 1,7 \mathrm{Cmolc} \mathrm{dm}-3 \mathrm{de} \mathrm{Mg} 2+; 18,6 \mathrm{Cmolc}$ dm-3 de K+; 583 mg dm-3 de P; $17 \mathrm{~g} \mathrm{~kg}^{-1}$ de N e 13,2\% de matéria orgânica. O adubo mineral de plantio foi de $334 \mathrm{~kg} \mathrm{ha}^{-1}$ de Ureia (67 kg no plantio e $267 \mathrm{~kg}$ em cobertura); $277 \mathrm{~kg} \mathrm{ha}^{-1}$ de Termofosfato magnesiano no plantio; $144 \mathrm{~kg} \mathrm{ha}^{-1} \mathrm{de}$ Cloreto de Potássio (28 kg ha ${ }^{-1}$ no plantio e $116 \mathrm{~kg} \mathrm{ha}^{-1} \mathrm{em}$ cobertura), conforme recomendações de Ribeiro et al. (1999).

Cada parcela era composta de $20 \mathrm{~m}^{2}(5 \mathrm{x} 4 \mathrm{~m})$. As plantas foram espaçadas de $1 \mathrm{x} 1 \mathrm{~m}$, totalizando 20 plantas por 
parcela e foram utilizadas as duas linhas centrais para análise de frutos. Utilizou-se a linhagem de pimenta dedo de moça amarela IFET 1572 (Capsicum baccatum), oriunda do Programa de Melhoramento Genético do IF Goiano - Campus Ceres.

Para o controle das plantas daninhas foram realizadas três capinas manuais. Não foi feita a aplicação de inseticidas ou fungicidas na cultura. O ciclo fenológico da pimenta foi de 210 dias após o transplante (DAT). As colheitas foram realizadas de 15 em 15 dias, totalizando seis colheitas, com início aos 95 dias após o transplante.

Os frutos foram colhidos quando se apresentavam maduros e avaliados os seguintes parâmetros: produtividade de frutos (PROD) em tha $\mathrm{er}^{-1}$; número de frutos por planta (NFP); peso de frutos (PF) em g; comprimento de frutos (COMP) em $\mathrm{mm}$; diâmetro de fruto (DIAM) em mm e espessura de polpa (ESP) em mm. As análises das variáveis seguiram a metodologia de análise quantitativa (Köche, 2011).

Para comparar as doses de adubo orgânico quanto à produtividade, calculou-se o índice de eficiência agronômica (IEA) (Goedert et al., 1986), de acordo com a fórmula: IEA $(\%)=[(\mathrm{Po}-\mathrm{Pt}) /(\mathrm{Pm}-\mathrm{Pt})]$ x 100; Onde: Po: produtividade obtida com o adubo em teste, na dose $\mathrm{n}$; Pt: produtividade do tratamento testemunha; Pm: produtividade obtida com o adubo mineral.

Os dados foram submetidos à análise de variância pelo teste $\mathrm{F}$ e para as doses de adubo orgânico foi feita a análise de regressão. Os ajustes dos modelos foram feitos com base na sua significância e o coeficiente de determinação (R2). O efeito médio das doses de adubo orgânico foi comparado com o tratamento de adubo mineral a partir do teste de Tukey a $5 \%$ $(\mathrm{p}<0,05)$ de probabilidade. As análises foram realizadas com o programa estatístico $\mathrm{R}$ ( $\mathrm{R}$ development core team, 2013).

\section{Resultados e Discussão}

Na Tabela 1 estão os resultados para o teste de Tukey para produtividade, número de frutos por planta, peso de frutos, comprimento e diâmetro de frutos e espessura de polpa de frutos de pimenta dedo de moça IFET 1572 sob adubo orgânico e mineral. Observa-se que para os tratamentos houve efeito significativo $(\mathrm{p} \leq 0,05)$ apenas sobre os resultados de produtividade $\mathrm{e}$ número de frutos por planta (Figuras 2 e 3, respectivamente).

Os tratamentos com dose de 15, 30, 45 e $60 \mathrm{t} \mathrm{ha}^{-1}$ de adubo orgânico e mineral apresentaram maiores produtividades e foram iguais estatisticamente entre si. Os tratamentos de 45 e $60 \mathrm{t} \mathrm{ha}^{-1}$ foram diferentes do tratamento controle (Zero). Os incrementos de produtividade dos tratamentos com adubo orgânico foram superiores ao adubo mineral em 33,47, 42,77, 59,24 e $54,54 \%$, respectivamente. 
Tabela 1. Produtividade de frutos (PROD), número de frutos por planta (NFP), peso de frutos (PF), comprimento de frutos (COMP), diâmetro de fruto (DIAM), espessura da polpa (ESP) de frutos de pimenta dedo de moça IFET 1572 submetidas as doses de adubo orgânico e mineral. Ceres, GO. 2017.

\begin{tabular}{ccccccc}
\hline $\begin{array}{c}\text { Tratamentos } \\
\mathrm{t} \mathrm{ha}^{-1}\end{array}$ & PROD & NFP & PF & COMP & DIAM & ESP \\
\hline 0 & $\mathrm{t} \mathrm{ha}^{-1}$ & un & $\mathrm{g}$ & $\mathrm{mm}$ & $\mathrm{mm}$ & $\mathrm{mm}$ \\
15 & $5,871 \mathrm{~b}$ & $66,62 \mathrm{~b}$ & $8,80 \mathrm{a}$ & $81,23 \mathrm{a}$ & $18,24 \mathrm{a}$ & $1,76 \mathrm{a}$ \\
30 & $9,319 \mathrm{ab}$ & $106,04 \mathrm{ab}$ & $8,79 \mathrm{a}$ & $81,72 \mathrm{a}$ & $19,38 \mathrm{a}$ & $1,72 \mathrm{a}$ \\
45 & $9,968 \mathrm{ab}$ & $105,25 \mathrm{ab}$ & $9,46 \mathrm{a}$ & $84,59 \mathrm{a}$ & $18,48 \mathrm{a}$ & $1,74 \mathrm{a}$ \\
60 & $11,118 \mathrm{a}$ & $113,75 \mathrm{a}$ & $9,72 \mathrm{a}$ & $80,88 \mathrm{a}$ & $18,72 \mathrm{a}$ & $1,80 \mathrm{a}$ \\
& $10,790 \mathrm{a}$ & $111,33 \mathrm{a}$ & $9,61 \mathrm{a}$ & $84,31 \mathrm{a}$ & $18,94 \mathrm{a}$ & $1,79 \mathrm{a}$ \\
Mineral & $6,982 \mathrm{ab}$ & $77,67 \mathrm{ab}$ & $8,65 \mathrm{a}$ & $83,74 \mathrm{a}$ & $18,52 \mathrm{a}$ & $1,68 \mathrm{a}$ \\
\hline C.V $(\%)$ & 22,91 & 19,82 & 6.98 & 3.3 & 3,83 & 4,71 \\
\end{tabular}

Nota. Médias seguidas por mesmas letras na coluna não diferem significativamente pelo teste de Tukey, a 5\% de probabilidade. Source: Authors.

No período do experimento ocorreu uma precipitação de $676,3 \mathrm{~mm}$ de chuva, que certamente, não foi suficiente para atender às necessidades da cultura. A partir do mês de março foram observados sintomas visuais de murcha nas plantas. Segundo Lima et al. (2013), esse fator pode limitar o rendimento da cultura, pois, a fase reprodutiva da planta é a mais sensível ao déficit hídrico. Os tratamentos controle e adubo mineral por não terem recebido adubo orgânico, podem ter apresentando maior déficit hídrico, uma vez que, o esterco bovino melhora a capacidade de infiltração e retenção de água, além de promover a maior eficiência no uso da água (Clemente et al., 2012; Maia Filho et al., 2013).

Provavelmente, a precipitação irregular ocorrida no período do experimento prejudicou a produtividade de pimentas. Barroca et al. (2015) ao avaliarem o efeito de lâminas de irrigação aplicadas por gotejamento em pimentas dedo de moça,

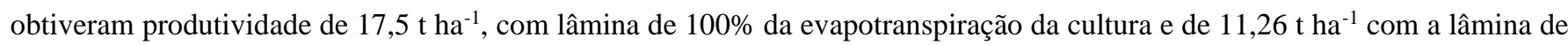
$40 \%$ da ETc. Nota-se que a pimenta dedo de moça responde a irrigação e há perdas conforme sofre déficit hídrico.

Os resultados de produtividade obtidos neste estudo estão próximos da mínima brasileira, que varia de $10 \mathrm{a} 25 \mathrm{t} \mathrm{ha}^{-1}$ (Pinheiro et al., 2012). Oliveira et al. (2014), ao trabalharem com fertilizante líquido obtiveram maior produtividade de pimenta dedo de moça de 13,84 $\mathrm{t} \mathrm{ha}^{-1}$ e média de 9,6 $\mathrm{t} \mathrm{ha}^{-1}$ entre os tratamentos estudados, em sistema irrigado por gotejamento. Os resultados obtidos pelos autores estão próximos ao do presente trabalho, onde, a média dos tratamentos com o adubo orgânico foi de $9,45 \mathrm{tha}^{-1}$.

Os resultados da produtividade de pimenta dedo de moça sob as doses crescentes de adubação orgânica submetidos à análise de regressão polinomial, revelou-se efeito significativo $(\mathrm{p} \leq 0,05)$ para uma função quadrática (Figura 2$)$. O coeficiente de determinação explica que o efeito dos tratamentos sobre a variável analisada foi na ordem de 96,76\% (Figura 2). De acordo com a derivada da função de produtividade obtida, o rendimento máximo $\left(11,18 \mathrm{t} \mathrm{ha}^{-1}\right)$ é atingido com aplicação de $48,15 \mathrm{t}^{-1}$ de esterco bovino. 
Figura 2. Produtividade de frutos de pimenta dedo de moça IFET 1572 sob doses de adubo orgânico no plantio.

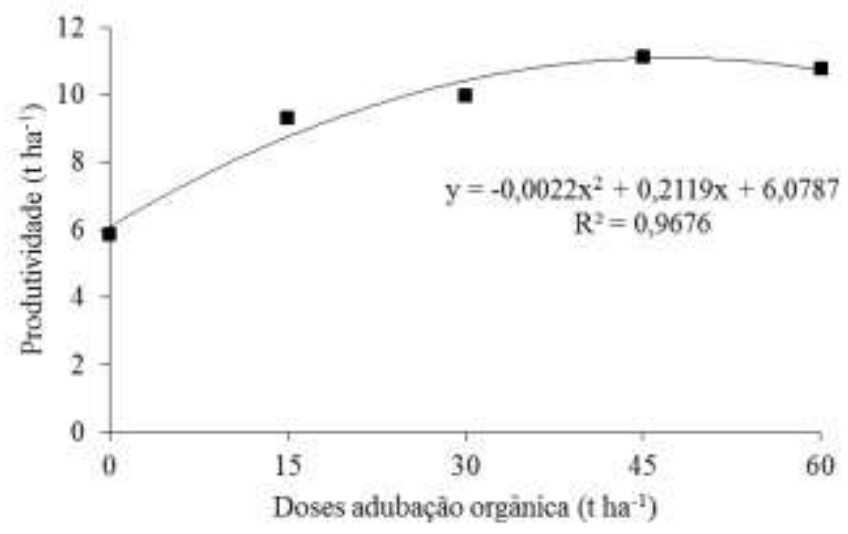

Fonte: Autores.

A dose de adubo orgânico que favorece a maior produtividade é maior que a quantidade de esterco recomendada pela Embrapa para a cultura, que é de $30 \mathrm{t} \mathrm{ha}^{-1}$ (Costa \& Henz, 2007). Os parâmetros de recomendação para a cultura da pimenta indicada pela Embrapa são para a cultura do pimentão (Ribeiro et al., 1999). Não há recomendação específica para a cultura de pimentas. Neste caso, há a necessidade de novos estudos para consolidar o uso de adubo orgânico na cultura de pimentas do gênero Capsicum.

Araújo et al. (2007), observaram que a dose de $28 \mathrm{t} \mathrm{ha}^{-1}$ de esterco bovino no cultivo de pimentão foi o suficiente para favorecer a produtividade máxima da cultura. Os autores observaram que doses superiores promoveram diminuição da produtividade. Assim, como encontrado no presente trabalho a produtividade tende a decrescer a partir de 48,15 t ha ${ }^{-1}$. Mendes et al. (2020) observaram que o esterco bovino associado ao pó de rocha pode ser uma opção para a produção de pimentões, com um efeito positivo do aumento do uso do esterco bovino nesta cultura.

Os resultados obtidos para número de frutos por planta foram iguais estatisticamente aos da produtividade (Tabela 1). O número de frutos por planta contribuiu para o resultado da produtividade de pimentas. Quanto maior foi o número de frutos de pimenta, maior foi a produtividade. As doses do adubo orgânico e mineral proporcionaram maior quantidade de frutos por planta de pimenta e foram diferentes estatisticamente do controle (Figura 3). Provavelmente, este efeito pode ter sido por causa da liberação de nutrientes de forma mais gradativa promovida pelo esterco bovino, que atende à demanda da cultura nos momentos em que a planta mais precisa para a produção (Oliveira et al., 2015; Souza \& Melo, 2003).

De acordo com o número de frutos obtidos, o rendimento máximo de frutos (115 frutos por planta) seria atingido com aplicação de 44,5 $\mathrm{t} \mathrm{ha}^{-1}$ de esterco bovino. 
Figura 3. Número de frutos por planta (NFP) de pimenta dedo de moça IFET 1572 sob doses de adubo orgânico no plantio.

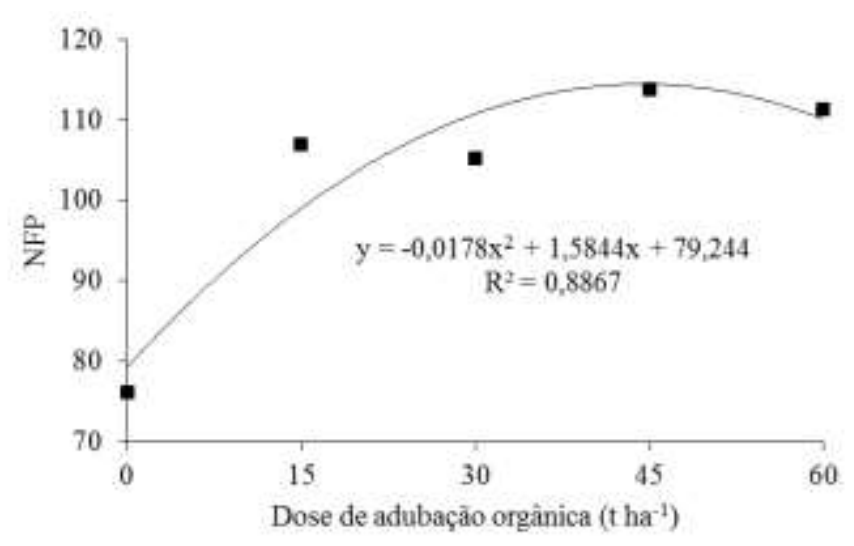

Fonte: Autores.

O resultado do Índice de Eficiência Agronômica (IEA) da pimenta dedo de moça quanto à produtividade do adubo orgânico, apresentou diminuição na seguinte ordem: $45 \mathrm{t} \mathrm{ha}^{-1}(228,98 \%)>30 \mathrm{tha}^{-1}(208,48 \%)>60 \mathrm{t} \mathrm{ha}^{-1}(192,03 \%)>15 \mathrm{t} \mathrm{ha}^{-1}$ $(130,52 \%)>$ Mineral $(100 \%)$. O IEA das doses de esterco bovino indica que esse fertilizante oferece boas perspectivas no uso em pimentas do gênero Capsicum. No caso do presente estudo, com base nos dados observados, a aplicação de $45 \mathrm{t} \mathrm{ha}^{-1} \mathrm{de}^{\mathrm{a}}$ esterco apresentou melhor IEA $(228,98 \%)$. Todas as doses obtiveram resultados superiores em relação ao adubo mineral. O uso de esterco bovino é uma opção que apresenta eficiência agronômica no que se refere a produtividade da cultura de pimenta em condição de sequeiro.

Os dados para as variáveis produção de frutos, comprimento, diâmetro e espessura da polpa dos frutos de pimenta dedo de moça apresentaram resultados iguais entre si para todos os tratamentos.

Como destaca Veras et al. (2019) o uso de adubos orgânicos quando comparado a adubação mineral, apresentam viabilidade de uso para culturas comerciais, sendo um material importante que pode ser usado na produção de olerícolas. Assim, como observado no presente estudo, a aplicação do esterco bovino curtido na cova de plantio pode substituir a adubação mineral sem comprometer o desempenho da cultura quando cultivado em sistema de sequeiro.

São poucos os estudos descritos na literatura do uso de esterco bovino na produção de pimentas, em particular na região Central do Brasil. Assim, para a produção com adubação orgânica para a agricultura familiar é de suma importância novos estudos de acordo com a realidade local.

\section{Conclusão}

As doses de adubo orgânico não proporcionaram diferença significativa na produtividade e no número de frutos por planta de pimentas. Os resultados foram maiores que no tratamento controle.

As doses de adubo orgânico de 45 e $60 \mathrm{t} \mathrm{ha}^{-1}$ proporcionam maiores produtividades de pimentas que o controle.

As doses de adubo orgânico e mineral não proporcionaram diferença significativa nas variáveis de produção, comprimento e diâmetro de frutos e espessura da polpa.

$\mathrm{O}$ maior índice de eficiência agronômica quanto à produtividade foi obtido na dose de $45 \mathrm{tha}^{-1}$ de adubo orgânico em relação ao adubo mineral.

O uso de esterco bovino pode ser indicado na produção de pimenta dedo de moça na região de Ceres, GO.

Estudos com diferentes opções de adubos orgânicos, métodos aplicação e efeito sobre a qualidade química e nutricional dos frutos são importantes a serem realizadas, afim de ter um conhecimento mais aprofundado do efeito dessa 
prática na qualidade dos frutos de pimenta dedo moça.

\section{Referências}

Alcântara, F. A. \& Ribeiro, C. S. C. Solos e adubação. In: Ribeiro, C. S. C., Lopes, A. C., Carvalho, S. I., Henz, G. P. \& ReifschneideR, F. J. B. (Ed.). Pimentas Capsicum. Embrapa Hortaliças, 2008. 81-94.

Barroca, M. V., Bonomo, R., Fernandes, A. A. \& Souza, J. M. (2015). Lâminas de irrigação nos componentes de produção das pimentas 'De cheiro' e 'Dedode-Moça. Revista Agro@mbiente Online, 9(3), 243-250. 10.18227/1982-8470ragro.v9i3.2342

Carvalho, S. I. C., Bianchetti, L. B., Bustamante, P. G. \& Silva, D. B. (2003). Catálogo de germoplasma de pimentas e pimentões (Capsicum spp.) da Embrapa Hortaliças. Embrapa Hortaliças, 49 p.

Clemente, R., Walker, D. J., Pardo, T., Martínez-Fernández, D. \& Bernal, M. P. (2012). The use of a halophytic plant species and organic amendments for the remediation of a trace elements contaminated soil under semiarid conditions. Journal of Hazardous Materials, 223-224(1), 63-71. 10.1016/j.jhazmat.2012.04.048

Costa, C. S. R. \& Henz, G. P. (2007). Pimenta (Capsicum spp.). Embrapa Hortaliças. Sistemas de Produção, 2. ISSN 1678-880x. Versão Eletrônica. Recuperado de: http://sistemasdeproducao.cnptia.embrapa.br/FontesHTML/Pimenta/Pimenta_capsicum_spp/cultivares.html.

Dias, V. V., Schultz, G., Schuster, M. S., Talamini, E. \& Révillion, J. P. (2015). O mercado de alimentos orgânicos: um panorama quantitativo e qualitativo das publicações internacionais. Ambiente \& Sociedade, 18(1), 155-174. 10.1590/1809-4422ASOC841V1812015en

Embrapa - Empresa Brasileira de Pesquisa Agropecuária. (2007). Sistemas de produção de pimentas (Capsicum spp). Embrapa Hortaliças, Sistemas de produção, 2. ISSN 1678-880x. Versão eletrônica. https://sistemasdeproducao.cnptia.embrapa.br/FontesHTML/Pimenta/Pimenta_capsicum_spp/index.html.

Embrapa - Empresa Brasileira de Pesquisa Agropecuária. (2013). Sistema brasileiro de classificação de solos. (3a ed.), Brasília, 353 p.

Freitas, R. A., Nascimento, W. M. \& Carvalho, S. I. C. (2008) Produção de sementes. In T. Ribeiro, C. S. C., Lopes, A. C., Carvalho, S. I., Henz, G. P. \& Reifschneider, F. J. B. (Ed.). Pimenta Capsicum. Brasília: Embrapa Hortaliças, 200 p.

Goedert, W., Souza, D. M. G. \& Thomaz, A. R. (1996). Princípios metodológicos para avaliação agronômica de fontes de fósforo. Embrapa.

Köche, J. C. (2011). Fundamentos de metodologia científica: teoria da ciência e iniciação à pesquisa. Editora Vozes.

Lima, E. M. C., Carvalho, J. A., Rezende, F. C., Thebaldi1, M. S. \& Gatto, R. F. (2013). Rendimento da pimenta cayenne em função de diferentes tensões de água no solo. Revista Brasileira de Engenharia Agrícola e Ambiental, 17(11), 1181-1187. 10.1590/S1415-43662013001100008

Lopes, J. D., Rodrigues, M. H. B. S., Hafle, O. M., Santos, V. M., Melo, E. N. \& Silva, J. N. (2020). Agronomic performance of the fig tree 'roxo de valinhos' submitted to different organic fertilizers. Research, Society and Development, 9(10), e2309108313. http://dx.doi.org/10.33448/rsd-v9i10.8313

Maia Filho, F. C. F., Mesquita, E. F., Guerra, H. O. C., Moura, M. F. \& Chaves, L. H. G. (2013). Effect of cattle manure on sunflower production and water use in two types of soil. Revista Ceres, 60(3), 397-405. 10.1590/S0034-737X2013000300013

Mendes, K. F., Vieira, H., Pereira Júnior, E. B., Moreira, J. N., Vale, K. S., Caiana, C. R. A., Bezerra Neto, D. C., Medeiros, A. C. \& Maracajá, P. B. (2020). Production pepper cultivated with stone dust and manure in semi-arid region. Research, Society and Development, 9(7), 1-15, e487974360. http://dx.doi.org/10.33448/rsd-v9i.4360

Oliveira, A. P., Gondim, P. C., Silva, O. P. R., Oliveira, A. N. P., Gondim, S. C. \& Silva, J. A. (2013). Produção e teor de amido da batata-doce em cultivo sob adubação com matéria orgânica. Rev. bras. eng. agríc. ambient., 17(8), 830-834. 10.1590/S1415-43662013000800005

Oliveira, J. R., Gomes, R. L. F., Araújo, A. S. F., Marini, F. S., Lopes, J. B. \& Araújo, R. M. (2014). Estado nutricional e produção da pimenteira com uso de biofertilizantes líquidos. Revista Brasileira de Engenharia Agrícola e Ambiental, 18(12), 1241-1246. 10.1590/1807-1929/agriambi.v18n12p1241-1246

Oliveira, M. L. J., Araujo, A. S. F. \& Melo, W. J. (2015). Chromium in soil organic matter and cowpea after four consecutive annual applications of composted tannery sludge. Revista Brasileira de Ciências do Solo, 39(1), 297-302. 10.1590/01000683rbcs20150158

Pinheiro, J. B., Amaro, g. B., Pereira, R. B. (2012). Nematóides em pimentas do gênero Capsicum. EMBRAPA. 9p.

Pinto, C. M., Puiatti, M., Caliman, F. R. B., Moreira, G. R., Mattos, R. N. (2006). Clima, época de semeadura, produção de mudas e espaçamento na cultura da pimenta. Informe Agropecuário: Cultivo da pimenta, 27(235), 40-49.

Ponisio, L. C., M'Gonigle, L. K., Mace, K. C., Palomino, J., Valpine, P. \& Kremen, C. (2014). Diversification practices reduce organic to conventional yield gap. Proceedings of the Royal Society B: Biological Sciences, 282(1799), 20141396-20141396. 10.1098/rspb.2014.1396

$\mathrm{R}$ Development Core Team. (2013). R: a language and environment for statistical computing. Vienna. http://softlibre.unizar.es/manuales/aplicaciones/r/fullrefman.pdf.

Reifschneider, F. J. B. (Org.). (2000). Capsicum: pimentas e pimentões no Brasil. Embrapa, 113 p.

Ribeiro, A. C., Guimarães, P. T. G. \& Alvarez, V. V. H. (Ed.). (1999). Recomendações para o uso de corretivos e fertilizantes em minas gerais: $5^{\text {a }}$ aproximação. Comissão de Fertilidade do Solo do Estado de Minas Gerais, 43-60.

Rodrigues, P. (2016). Pimenta: de todos sabores e gostos. A lavoura, ano 119(716), 9-25. https://www.embrapa.br/documents/1355126/10765216/2 016_11_01+Revista+A+Lavoura+-+Pimenta+\%28NCO+via+AE\%29.pdf/cb54ee6c-6de0-4a4d-ab8c-1137a38a05f9. 
Research, Society and Development, v. 10, n. 4, e10610413893, 2021

(CC BY 4.0) | ISSN 2525-3409 | DOI: http://dx.doi.org/10.33448/rsd-v10i4.13893

Silva, V. F., Bezerra, C. V. C., Nascimento, E. C. S., Ferreira, T. N. F., Lima, V. L. A., \& Andrade, L. O. (2019). Production of chili pepper under organic fertilization and irrigation with treated wastewater. Revista Brasileira de Engenharia Agrícola e Ambiental, 23(2), 84-89. 10.1590/18071929/agriambi.v23n2p84-89

Souza, W. J. O. \& Melo, W. J. (2003). Matéria orgânica de um Latossolo submetido a diferentes sistemas de produção de milho. Revista Brasileira de Ciência do Solo, 27(6), 1113-1122. 10.1590/S0100-06832003000600016

Vazquez-Vazquez, C., García-Hernández, J. L, Salazar-Sosa, E., Murillo-Abador, B., Orona-Castillo, I., Zúñiga-Tarango, R., Rueda-Puente, E. O. \& Preciado-Rangel, P. (2010). Rendimiento y valor nutritivo de forraje de alfalfa (Medicago sativa L.) con diferentes dosis de estiércol bovino. Mexicana Journal of Animal Science, 1(4), 363-372. http://www.scielo.org.mx/scielo.php?script=sci_arttext\&pid=S2007-11242010000400005\&lng=es.

Veras, R. N. S., Lima, D. S., Carvalho, J. A., Reis, A. S. \& Silva, M. R. Development evaluation of alface Vanda in relation to the use of chemical fertilizer and organic compost. Research, Society and Development, 8(1), e3581618. http://dx.doi.org/10.33448/rsd-v8i1.618

Zavattaro, L., Bechini, L., Grignani, C., Evert, F. V., Mallast, J., Spiegel, H., Sandén, T., Pecio, A., Cervera, J. V. G., Guzmán, G., Vanderlinden, K., D’horse, T., Ruysschaert, G. \& Hein, F. M. (2017). Agronomic effects of bovine manure: A review of long-term European field experiments. European Journal of Agronomy, 90, 127-138. 10.1016/j.eja.2017.07.01 